\title{
Frequency and form as determinants of functor sensitivity in English-acquiring infants
}

\author{
Rushen Shi \\ Département de psychologie, Université du Québec à Montréal, C.P.8888, Succursale Centre-Ville, Montréal, \\ Québec H3C 3P8 Canada \\ shi.rushen@uqam.ca \\ Anne Cutler \\ Max Planck Institute for Psycholinguistics, P.O. Box 310, 6500 AH Nijmegen, The Netherlands \\ Anne.Cutler@mpi.nl \\ Janet Werker and Marisa Cruickshank \\ Department of Psychology, University of British Columbia, 2136 West Mall, Vancouver, \\ British Columbia V6T1Z4, Canada \\ jwerker@psych.ubc.ca,marisa.cruick@gmail.com
}

\begin{abstract}
High-frequency functors are arguably among the earliest perceived word forms and may assist extraction of initial vocabulary items. Canadian 11- and 8-month-olds were familiarized to pseudo-nouns following either a high-frequency functor the or a low-frequency functor her versus phonetically similar mispronunciations of each, $k u h$ and ler, and then tested for recognition of the pseudo-nouns. A preceding the (but not kuh, her, ler) facilitated extraction of the pseudo-nouns for 11-month-olds; the is thus well-specified in form for these infants. However, both the and kuh (but not her-ler) facilitated segmentation for 8-month-olds, suggesting an initial underspecified representation of high-frequency functors.

(C) 2006 Acoustical Society of America

PACS numbers: 43.71.Ft $[\mathrm{JH}]$

Date Received: January 11, 2006 Date Accepted: March 27, 2006
\end{abstract}

\section{INTRODUCTION}

The production of an infant's first words is an achievement crowning months of perceptual work. One of the crucial perceptual tasks is the recognition of different acoustic realizations of a word in continuous speech. The speech which infants hear in the first year consists largely of continuous multi-word utterances, ${ }^{1}$ in which word boundaries are not reliably marked and in which words may appear in varying phonetic environments. Nonetheless, during the first year of life infants gradually attune perception to the native phonemic repertoire, ${ }^{2}$ acquire knowledge of acceptable versus unacceptable sequences of these sounds, ${ }^{3}$ and become sensitive to the probable patterns of words of their language. ${ }^{4}$ Construction of a vocabulary crucially depends on the ability to extract word forms from a continuous context. With a two-phase familiarization and test procedure, Jusczyk and Aslin ${ }^{5}$ demonstrated this extraction ability in prelinguistic infants; with the same type of procedure, the cortical responses to the occurrence in continuous speech of a form previously heard in isolation were observed. ${ }^{6}$ Since the first demonstration of noun segmentation, ${ }^{5}$ many subsequent studies ${ }^{7}$ have examined numerous aspects of content word segmentation.

Function words offer - at least in English and similar languages - a possible aid to infants in this task. More than half of English spontaneous speech consists of functors (e.g., determiners and conjunctions). ${ }^{8}$ There are few functor types, so the frequency with which each occurs is high. The high frequency of functors facilitates grammar acquisition in adults' learning of artificial languages. ${ }^{9}$ We here inquire whether they may also facilitate, early in language acquisition, the segmentation of potential vocabulary items from speech. 
Certainly infants are acquiring sensitivity to functors in the period in which they are beginning to amass a vocabulary. Behavioral ${ }^{10,11}$ and ERP ${ }^{12}$ evidence indicates that Englishlearning 10.5- to 11-month-old infants distinguish real from nonsense functors in continuousspeech contexts. Furthermore, infants at 11 and 13 months prefer real functors over minimally modified nonsense functors, suggesting that they recognize real functors with phonetically detailed representation. ${ }^{11}$ German-learning 7 - to 9 month-old infants ${ }^{13}$ and French-learning 6- to 8-month-old infants ${ }^{14}$ are able to segment function words from continuous speech. Note that German and French functors are phonologically stronger than English functors, which are predominantly weak. ${ }^{15}$ However, across typologically different languages infant-directed speech exhibits contrasting cues, acoustic as well as distributional, which support differentiation of functional from lexical elements. ${ }^{16}$

To examine whether functors facilitate segmentation of potential vocabulary items, we exploited the familiarity effects which appear in the two-phase familiarization and test procedures introduced into infant speech perception research by Jusczyk and Aslin. ${ }^{5}$ Infants express familiarity by listening longer in the test phase to known elements, usually items first presented in the familiarization phase. In a variation on this procedure we made familiarity dependent upon infants' ability to segment test items from a preceding functor. If infants are able to perform this segmentation, they will perceive the items presented in isolation in the test phase as familiar. However, if no segmentation occurs (i.e., functor and test item are perceived as an unsegmented whole), the test item when presented alone will be perceived as an unfamiliar form.

As described above, earlier work has shown that 11-month-olds can recognize the correct phonetic form of function words; we therefore began our study with 11-month-olds. Infants were first familiarized with pseudo-nouns preceded by either real or nonsense functors. In the test phase, they heard the same pseudo-nouns without the preceding context. As functor frequency is crucially involved in functors' facilitatory role in language acquisition, ${ }^{9}$ and as frequently encountered forms may prove particularly useful in segmentation, ${ }^{17}$ we reasoned that it would be important also to examine the frequency factor within the functor class. We therefore contrasted a high-frequency functor (the) with a functor with a lower frequency of occurrence (her). The difference between these two in the relevant speech type-speech to infants-was established in several corpora. In a corpus of 290094 words (word types $=50855)^{18}$ of 11 parents speaking to their infants (aged 9 to 13 months; each recorded bi-weekly) the frequency of the was 8513, and of her was 307. Parental speech to infants up to 24 months of age in three further corpora ${ }^{19-21}$ yielded a total of 71978 word tokens and 16310 word types; in this total, the token count for the is 2484 and for her 114. Thus although all functor elements have relatively high frequency compared with content words, the is likely to have occurred far more often than her in the listening experience of infants.

\section{EXPERIMENT 1}

Twenty-four 11-month-old monolingual English-learning infants completed the task, 8 in the high frequency and 16 in the low frequency condition. Another 10 infants were tested but were excluded due to fussiness, equipment errors, or insufficient looking time during familiarization. Stimuli were the English functors the (high-frequency) and her (low-frequency), plus prosodically matched nonsense functors $k u h$ and ler, which differed from the and her only in the onset consonants. Stimuli were analyzed for duration, amplitude, and pitch to ensure they were well matched. Both her and ler were produced with a rhotic $/ \gamma /$ distinct from the schwa in the and $k u h$. Both types of functors were paired with the pseudo-nouns breek [brik] and tink [tink] to form 8 simple noun phrases, the breek, her tink, ler tink, etc. A native speaker of American English, a mother of a 6-month-old infant and naive as to the goal of the study, produced the stimuli in an infant-direct speech style. The final set of stimuli included three tokens of each of the eight sequences, and tokens of breek (15) and tink (15) in isolation.

During the familiarization phase, infants heard six alternating trials $(3+3)$ of "functor+breek" and "nonsense functor+tink" (or "functor+tink" and "nonsense functor +breek"). They were then immediately presented with four test trials (two trials with isolated 
breek tokens alternating with two trials of isolated tink tokens). All trials were $16 \mathrm{~s}$. The interstimulus duration between sequences was $500 \mathrm{~ms}$, with minor adjustments to maintain the same trial length despite slight durational variations among sequences. Orders and functorpseudoword pairing were counterbalanced across infants, but the test order was constrained: if the last familiarization sequence contained breek, the first test trial began with tink, and vice versa.

In a sound-attenuated chamber the infant sat on his/her parent's lap facing a central TV monitor and a loudspeaker. During trials, auditory stimuli were presented together with a blackand-white checkerboard on the screen, using HABIT $2000 .{ }^{22}$ The parent heard masking music over headphones. The researcher in the next room, blind to the trial orders, observed the infant through a closed-circuit TV, and pressed a computer key whenever an eye fixation occurred. Each trial was initiated upon the infant's eye fixation. Once a trial was initiated, it continued for the entire trial length. All looks to the monitor were recorded online, and were subsequently rescored from videotape records at 30 frames/s; statistical analyses were based on the offline data. Infants who did not reach $15 \mathrm{~s}$ of cumulative looking time while receiving the 48-s exposure of each sequence type during familiarization were excluded from the analysis, to ensure that all infants gave sufficient attention to the sequences before the test phase.

The comparison of interest is relative looking time to the display during presentation of each test pseudo-noun. If the first syllable (functor type) of the familiarization sequences is recognized as familiar, the pseudo-noun with which it is paired will be apprehended as a separate word form and will invoke longer looking time when it appears in isolation in the test phase.

Figure 1(a) shows the very clear pattern which appeared in this experiment. Pseudonouns which had been preceded by the in the familiarization phase elicited longer looking times than pseudo-nouns preceded by any other of the functor types. For each infant, we calculated the total looking time across breek and tink trials separately, and conducted a mixed $2 \times 2$ ANOVA with familiarization frequency (high, i.e., the-kuh, versus low, i.e., her-ler) as betweensubjects factor, and familiarization functor type (real, i.e., the-her, versus nonsense, i.e., kuhler) as within-subjects factor. This revealed no significant main effect of frequency, but a significant main effect of functor type, $F(1,22)=8.038 ; p=0.01$ (Mean=16.96 s, SE $=1.36 \mathrm{~s}$ for pseudo-nouns previously paired with real functors; Mean $=15.21 \mathrm{~s}, \mathrm{SE}=1.24 \mathrm{~s}$ for pseudonouns previously paired with nonsense functors); further, the interaction between these factors was significant: $F(1,22)=10.139 ; p=0.004$. Follow-up paired $t$-tests showed that infants listened significantly longer to pseudo-nouns previously familiarized with the (Mean $=19.41 \mathrm{~s}$, $\mathrm{SE}=2.18 \mathrm{~s}$ ) than to those previously familiarized with $k u h$ (Mean=15.69 s, SE=2.27 s), $t(7)$ $=3.9 ; p=0.006,2$-tailed. Every infant in this condition looked longer to the than to kuh nouns. In contrast, no difference was found in the low-frequency her-ler condition.

Infants' looking times during familiarization were analyzed in high- and lowfrequency conditions; there was no difference between real versus functor sequences, and familiarization exposure was equivalent across the two frequency conditions.

The test phase results clearly indicate that the high-frequency real functor the allows 11-month-old English-acquiring infants to segment adjacent word forms from it. Less frequent real functors, and nonsense functors, are significantly less effective. Note that the highfrequency group, in which the pattern is statistically robust, had only half as many participants as the low-frequency group. Thus we can be confident that the failure to find a significant realfunctor preference in the low-frequency group is not simply due to lack of power.

In Experiment 2 we repeated the task with younger listeners. In speech to 8-month-olds, the is also presumably the most frequent functor type. If frequency is the only determinant of the emerging sensitivity to functor identity, the may be equally effective for 8 -month-olds. 


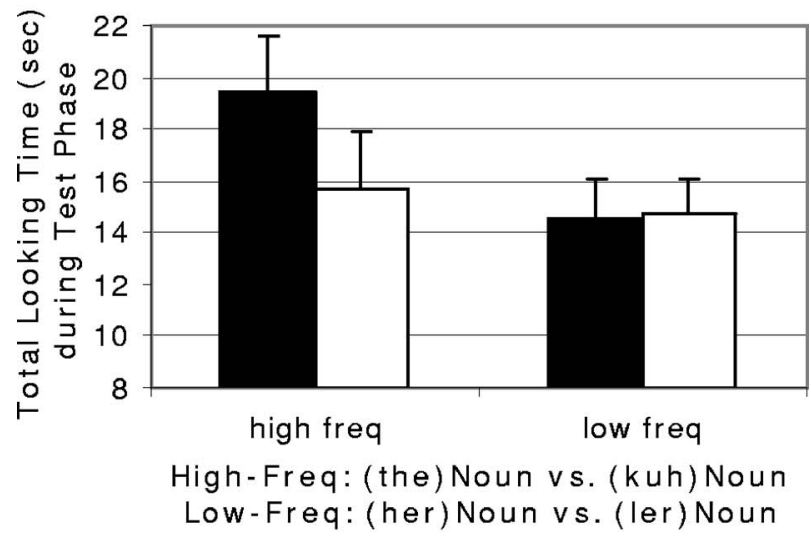

(functor) Noun $\square$ (nonsense) Noun

(a)

\section{8 -Month-Old Infants}

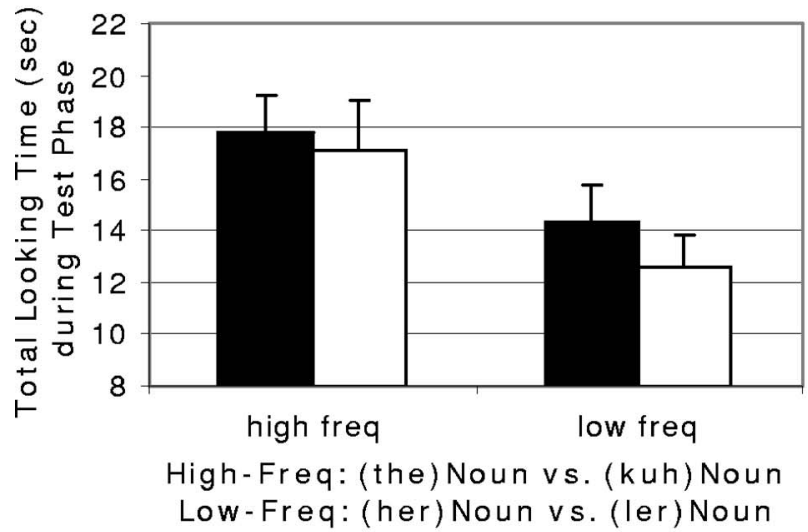

(functor) Noun $\square$ (nonsense) Noun

(b)

Fig. 1. 11-month-old [(a), Experiment 1] and 8-month-old [(b), Experiment 2] infants' mean total looking time (with SEs) to pseudo-nouns in the test phase, as a function of familiarization functor type. In the high-frequency condition one noun was heard with the and another noun with $k u h$ during familiarization, i.e., $($ the $)$ Noun, $(k u h)$ Noun. In the low-frequency condition the same nouns were heard with her and ler, respectively, during familiarization, i.e., (her)Noun, (ler)Noun.

\section{EXPERIMENT 2}

Twenty-four 8-month-old English-learning infants completed the task. Another five infants were excluded from data analyses due to fussiness, equipment failure, experimenter errors, or insufficient looking time during familiarization. Stimuli and design were as in Experiment 1.

Looking times during familiarization exposure to real versus nonsense functor sequences were compared; there were again no differences across the high- or low-frequency conditions. 
The test data were analyzed as a function of familiarization condition, as in Experiment 1 . There was no significant main effect of functor type and no interaction of functor type and frequency. However, as shown clearly in Fig. 1(b), there was a significant main effect of frequency: Looking time for pseudo-nouns was longer after familiarization with highfrequency than with low-frequency pairs $(F(1,22)=4.56, p=0.044$; Mean=17.43 s, SE $=1.52 \mathrm{~s}$ for pseudo-nouns previously paired with the or $k u h$; Mean=13.46 s, SE=1.07 s for pseudo-nouns previously paired with her or ler). In other words, when the data were collapsed across the and kuh, there was evidence of facilitation in the younger listeners' segmentation of the strings whereby the second element produced longer listening times in the test phase. No such effect was exercised by the lower-frequency pair her and ler, which differed by less than $2 \mathrm{~s}$ from each other and by more than $2 \mathrm{~s}$ from the-kuh.

\section{GENERAL DISCUSSION}

Our study has revealed that functors indeed assist young infants in segmenting potential word forms from continuous speech. The test words in this experiment were novel forms which the infants could not have already known. The infants were not yet active communicators; few 11-month-olds intentionally produce known words, and no 8-month-olds do. During the second half of their first year, though, infants are actively engaged in learning how to amass a stock of vocabulary items. The occurrence of function words can, we have shown, help them in this task. Constructing a memory representation of words in the mental lexicon requires first segmenting word-size chunks from longer utterances. The presence of a highly frequent, familiar functor assists the infants in treating the adjacent element as a separate word chunk.

Our results suggest it is not the syntactic function of the functors per se which exercises the helpful effect. It is, rather, the fact that they occur very frequently in speech (including infant-directed speech). In our study, the longest looking times were recorded to test pseudowords which had been presented with preceding the, the most frequently occurring word in all written and spoken frequency counts of the English language. This functor appears to have occurred often enough in the infants' listening experience such that whenever it is heard, the speech material following it is segmented from the and treated as a candidate vocabulary item. Clearly, very high frequency is necessary for this effect, because the real functor her, although in absolute terms also a frequently occurring English word, does not facilitate segmentation in the same way either for 11- or for 8-month-olds. As our tallies of word frequency in speech input to infants showed, infants hear her very much less often than the. The facilitatory effect in segmentation is restricted, at this age, to items which occur very frequently indeed.

Besides the effect of frequency, we discovered a further effect which we would interpret as an effect of acoustic form. For 8-month-olds, not only the but also its near match kuh facilitated segmentation and thereby produced longer looking times to the pseudo-words presented in isolation. As earlier work had shown, ${ }^{11} 8$-month-olds cannot distinguish real from nonsense functors during word recognition; the earliest age at which English functor representation in phonetic detail is established is $10.5-11$ months. ${ }^{10-12}$ It is thus no surprise to find that 8-month-olds do not distinguish the from $k u h$ in this word segmentation task. What is surprising is that both the and kuh act as significantly more effective facilitators of segmentation for this age group than do her and ler. Our interpretation of this effect is that for the 8-month-olds, a representation of the highest-frequency form is already in place, but it is as yet acoustically underspecified. Since we only compared the with $k u h$, and in particular we did not test the other frequent functor $a$, we cannot make a precise statement about the range of forms which would satisfy this underspecified representation (any syllable with schwa, schwa plus any singleton onset, only acoustically weak onsets, etc.); however, the failure of her and ler to exercise the facilitatory effect suggests that the form of the underspecified representation has to be reasonably close to the acoustic form of the itself.

The difference between full vowels and reduced vowels (mostly schwa) is extremely salient in English; listeners find it acceptable to replace any full vowel by the same vowel with any other level of stress (e.g., the first syllables of audience, auditorium, and audition, with primary, secondary, and no stress, respectively, may be interchanged), but never tolerate full 
vowels exchanging with reduced (e.g., the first syllable of addition). ${ }^{23}$ Newborn infants are already capable of distinguishing between lists of English content words (which all must have at least one full syllable) and function words (which mostly consist of a monosyllable with a reduced vowel). ${ }^{24}$ We suggest that our results indicate that this full-reduced distinction is useful to infants in the crucial work of acquiring a vocabulary. The frequent occurrence of functors with a reduced vowel (not only the but also $a$ ) allows for the early construction of an underspecified "functor" representation with a reduced vowel, and this underspecified representation serves to facilitate segmentation of potential vocabulary items from speech input. At 8 months, this is a useful aid in the beginnings of vocabulary acquisition. Accumulation of further experience makes formation of phonetically detailed functor representations possible; thus at 11 months, the facilitates segmentation but kuh no longer does. Our previous study ${ }^{11}$ showed that a wider range of functors is represented in phonetic detail by age 13 months, so that we would predict that 13-month-old listeners in a two-phase experiment would be able to use her also in segmentation.

The task of bootstrapping a vocabulary from continuous speech input is a formidable one, but infants begin to accomplish it by the end of their first year of life. Our results shed further light on how they do this. Frequently occurring forms, perhaps at first represented without full specification, serve as a framework against which potential candidates for vocabulary membership may be identified and extracted.

\section{ACKNOWLEDGMENTS}

This research was funded by grants from NSERC and FQRSC to R.S., and NSERC and McDonnell grants to J.W., R.S. and J.W. also acknowledge support from Max Planck Society visiting research stipends. Partial reports of this work were presented at the XIVth and XVth Biennial International Conference on Infant Studies.

${ }^{1}$ J. van de Weijer, "Language input for word discovery," Ph.D. dissertation, University of Nijmegen, Nijmegen 1998.

${ }^{2}$ J. Werker and R. Tees, "Cross-language speech perception: Evidence for perceptual reorganization during the first year of life," Infant Behav. Dev. 7, 49-63 (1984).

${ }^{3}$ P. Jusczyk, J. Charles-Luce, and P. Luce, "Infants' sensitivity to phonotactic patterns in the native language," J. Mem. Lang. 33, 630-645 (1994).

${ }^{4}$ P. Jusczyk, A. Cutler, and N. Redanz, "Infants' preference for the predominant stress patterns of English words," Child Dev. 64, 675-687 (1993).

${ }^{5}$ P. Jusczyk and R. Aslin, "Infants' detection of the sound patterns of words in fluent speech," Cogn. Psychol. 23, 1-23 (1995).

${ }^{6}$ V. Kooijman, P. Hagoort, and A. Cutler, "Electrophysiological evidence for prelinguistic infants' word recognition in continuous speech," Cogn. Brain Res. 24, 109-116 (2005).

${ }^{7}$ J. Saffran, J. Werker, and L. Werner, "The infants's auditory world: Hearing, speech, and the beginnings of language," in Handbook of Child Psychology, 6th ed., edited by D. Kuhn and M. Siegler (Wiley, Englewood Cliffs, NJ, 2006).

${ }^{8}$ A. Cutler and D. Carter, "The predominance of strong initial syllables in the English vocabulary," Comput. Speech Lang. 2, 133-142 (1987).

${ }^{9} \mathrm{~V}$. Valian and S. Coulson, “Anchor points in language learning: The role of marker frequency,” J. Mem. Lang. 27, 71-86 (1988).

${ }^{10}$ M. Shady, "Infants' sensitivity to function morphemes," Ph.D. thesis, SUNY, Buffalo, 1996.

${ }^{11}$ R. Shi, J. Werker, and A. Cutler, "Recognition and representation of function words in English-learning infants," Infancy (in press).

${ }^{12}$ V. Shafer, J. Shucard, D. Shucard, and L. Gerken, “An electrophysiological study of infants' sensitivity to the sound patterns of English speech,” J. Speech Hear. Res. 41, 874-886 (1998).

${ }^{13}$ B. Höhle and J. Weissenborn, “German-learning infants' ability to detect unstressed closed-class elements in continuous speech,” Dev. Sci. 6, 122-127 (2003).

${ }^{14}$ R. Shi, A. Marquis, and B. Gauthier, "Segmentation and representation of function words in preverbal Frenchlearning infants," in Proceedings of the 30th Boston University Conference on Language Development (Cascadilla, Somerville, MA, 2006).

${ }^{15}$ A. Cutler, "Phonological cues to open- and closed-class words in the processing of spoken sentences," J. Psycholinguist. Res. 22, 109-131 (1993).

${ }^{16} \mathrm{R}$. Shi, J. Morgan, and P. Allopenna, "Phonological and acoustic bases for earliest grammatical category assignment: A cross-linguistic perspective," J. Child Lang. 25, 169-201 (1998).

${ }^{17}$ H. Bortfeld, J. Morgan, R. Golinkoff, and K. Rathbun, "Mommy and Me: Familiar names help launch babies into speech stream segmentation," Psychol. Sci. 16, 298-304 (2005). 
${ }^{18} \mathrm{M}$. Brent and J. Siskind, "The role of exposure to isolated words in early vocabulary development," Cognition $\mathbf{8 1}$, B33-44 (2001).

${ }^{19}$ E. Bates, I. Bretherton, and L. Snyder, From First Words to Grammar (Cambridge University Press, New York, 1988).

${ }^{20} \mathrm{~N}$. Bernstein-Ratner and C. Pye, "Higher pitch in babytalk is not universal: Acoustic evidence from Quiche Mayan,” J. Child Lang. 11, 515-522 (1984).

${ }^{21}$ R. Higginson, "Fixing-assimilation in language acquisition," Ph.D. thesis, Washington State University, 1985.

${ }^{22}$ L. Cohen, D. Atkinson, and H. Chaput, "Habit 2000: A new program for testing infant perception and cognition," [Computer software]. Austin, the University of Texas, 2000.

${ }^{23}$ B. Fear, A. Cutler, and S. Butterfield, "The strong/weak syllable distinction in English," J. Acoust. Soc. Am. 97, 1893-1904 (1995).

${ }^{24}$ R. Shi, J. Werker, and J. Morgan, "Newborn infants' sensitivity to perceptual cues to lexical and grammatical words," Cognition 72, B11-21 (1999). 